\title{
Do We Initiate Chemotherapy at an Early Stage of Hormone-Sensitive Metastatic Prostate Cancer?
}

\author{
Erdinç NAYIR, ${ }^{1}$ Özge KESKIN² \\ 'Department of Medical Oncology, Kahramanmaraş Necip Fazıl City Hospital, Kahramanmaraş-Turkey \\ ${ }^{2}$ Department of Medical Oncology, Aydın Atatürk State Hospital, Aydın-Turkey
}

Dear Editor;
Prostate cancer is the most frequently seen type of cancer in men. It is the second most frequent cause of mortality.[1] In hormone-sensitive metastatic prostate adenocarcinoma, standard treatment approach is androgen deprivation therapy (ADT). ADT, orchiectomy or LHRH analogues are used singly or in combination Addition of chemotherapy to standard treatment at an early stage has been investigated in three important studies (GETUG-AFU 15, CHAARTED, STAMPEDE).[2]

In the GETUG-AFU 15, study patients with metastatic prostate adenocarcinoma have been divided into two arms. One arm received ADT, and the other arm received 9 cycles of docetaxel therapy in addition to ADT. During a 50 years of median follow-up overall survival (OS) in the ADT-docetaxel arm was 58.9 months, and in the ADT arm, 54.2 months (HR 1.01, $\mathrm{p}=0.955$ ). Biochemical progression-free survival (PFS) was 22.9 months in the ADT-docetaxel arm $(p=0.005)$ arm, and 12.9 months in the ADT arm. Clinical PFS was 23.5 months in the ADT - docetaxel arm, and 15.4 months in the ADT arm ( $\mathrm{p}=0.015)$. During 80 months of median follow-up OS was 60.9 months in the ADTdocetaxel arm, and 35.1 months in the ADT arm which was significantly different between groups. However in high-volume disease (visceral metastases or $\geq 4$ bone lesions with $\geq 1$ beyond the vertebral bodies and pelvis) any difference was not detected. Adverse effects as neutropenia, and neutropenic fever $(8 \%)$, nausea, vomiting, and neuropathy were more frequently seen in the ADT - docetaxel arm. [3]
In the CHAARTED study, patients with metastatic prostate cancer were divided into 2 arms. One arm received 6 cycles of docetaxel together with ADT, and the other arm received ADT. During 28.9 months of median follow-up period OS was 57.6 months in the ADT-docetaxel arm, and 44 months in the ADT arm (HR 0.61, $\mathrm{p}<0.001$ ). OS was detected as 49.2 months in the high-volume disease in the combination arm, and 32.2 months in the ADT arm (HR 0.60, $\mathrm{p}<0.001)$ Time to clinical progression was 33 months in the combination arm, and 19.8 months in the ADT arm (HR 0.61, $\mathrm{p}<0.001)$. Neutropenic fever was more frequently seen (6.2\%) in the combination arm.[4]

However STAMPEDE study was more heterogenous when compared with other studies.Not only patients with metastatic prostate cancer, but those with locallyadvanced disease were also included in the study. Sixtytwo percent of the patients had metastatic disease. The patients were divided into 4 arms as follows: ADT, ADT - Zolendronic acid, ADT - Docetaxel, and ADT - Zolendronic acid - Docetaxel. During median follow-up period of 43 months, OS was 67 months in the ADT docetaxel arm, and 77 months in ADT arm $(\mathrm{p}=0.003)$. However PFS was 37 months in the ADT - docetaxel arm, and 21 months in the ADT arm $(\mathrm{p}=0.0001)$. However in the metastatic subgroup OS was 43 months in the ADT arm, and 65 months in the ADT-docetaxel arm $(\mathrm{p}=0.002)$. As detected in subgroup analysis,patients aged $<70$ years with metastatic disease, and Gleason score of $\leq 7$ benefited more in the combination arm. In this study neutropenic fever was more frequently seen (15\%) in the docetaxel containing arms.[5]
Received: October 17, 2016

Accepted: December 16, 2016

Online: January 17, 2017

Accessible online at:

www.onkder.org
Dr. Erdinç NAYIR

Necip Fazıl Şehir Hastanesi ,

Tıbbi Onkoloji Anabilim Dalı,

Kahramanmaraş-Turkey

E-mail: drerdincnyr@gmail.com 
In a meta-analysis encompassing these three studies, docetaxel given with ADT created a significant difference in OS, and PFS rates. A significant difference was not detected between high (HR 0.67), and low-volume (HR 0.80) diseases ( $p=0.53$ ). Addition of docetaxel to ADT decreased mortality risk $27 \%$ in metastatic (HR 0.73), and 33\% in high-volume metastatic disease (HR 0.67).[6]

In conclusion, in hormone- sensitive metastatic prostate adenocarcinoma, addition of docetaxel to ADT is an effective approach. Although the number of bone lesions in high-volume disease is debatable, fit patients younger than 70 years of age with visceral metastasis appear to get higher benefit from this combination treatment. It should not be forgotten that application of this combination will increase the risk of febrile neutropenia.

\section{References}

1. Siegel RL, Miller KD, Jemal A. Cancer statistics, 2016. CA Cancer J Clin 2016;66(1):7-30. Crossret

2. Bernard B, Sweeney CJ. Management of metastatic hormone-sensitive prostate cancer. Curr Urol Rep 2015;16(3):14. Crossret

3. Gravis G, Fizazi K, Joly F, Oudard S, Priou F, Esterni $\mathrm{B}$, et al. Androgen-deprivation therapy alone or with docetaxel in non-castrate metastatic prostate cancer (GETUG-AFU 15): a randomised, open-label, phase 3 trial. Lancet Oncol 2013;14(2):149-58. Crossree

4. Sweeney CJ, Chen YH, Carducci M, Liu G, Jarrard DF, Eisenberger M, et al. Chemohormonal Therapy in Metastatic Hormone-Sensitive Prostate Cancer. N Engl J Med 2015;373(8):737-46. Crossre

5. James ND, Sydes MR, Clarke NW, Mason MD, Dearnaley DP, Spears MR, et al. Addition of docetaxel, zoledronic acid, or both to first-line long-term hormone therapy in prostate cancer (STAMPEDE): survival results from an adaptive, multiarm, multistage, platform randomised controlled trial. Lancet 2016;387(10024):1163-77. Crossret

6. Tucci M, Bertaglia V, Vignani F, Buttigliero C, Fiori C, Porpiglia F, et al. Addition of Docetaxel to Androgen Deprivation Therapy for Patients with Hormone-sensitive Metastatic Prostate Cancer: A Systematic Review and Meta-analysis. Eur Urol 2016;69(4):563-73. Crossre 